\title{
Spinodal decomposition in fine grained materials
}

\author{
H RAMANARAYAN and T A ABINANDANAN* \\ Department of Metallurgy, Indian Institute of Science, Bangalore 560 012, India
}

\begin{abstract}
We have used a phase field model to study spinodal decomposition in polycrystalline materials in which the grain size is of the same order of magnitude as the characteristic decomposition wavelength $\left(\boldsymbol{\lambda}_{\mathrm{SD}}\right)$. In the spirit of phase field models, each grain $(i)$ in our model has an order parameter $\left(\eta_{i}\right)$ associated with it; $\eta_{i}$ has a value of unity inside the $i$ th grain, decreases smoothly through the grain boundary region to zero outside the grain. For a symmetric alloy of composition, $c=0-5$, our results show that microstructural evolution depends largely on the difference in the grain boundary energies, $\gamma_{\mathrm{gb}}$, of A-rich $(\alpha)$ and B-rich ( $\beta$ ) phases. If $\gamma_{\mathrm{gb}}^{\alpha}$ is lower, we find that the decomposition process is initiated with an $\alpha$ layer being formed at the grain boundary. If the grain size is sufficiently small (about the same as $\lambda_{\mathrm{SD}}$ ), the interior of the grain is filled with the $\beta$ phase. If the grain size is large (say, about $10 \lambda_{S D}$ or greater), the early stage microstructure exhibits an A-rich grain boundary layer followed by a B-rich layer; the grain interior exhibits a spinodally decomposed microstructure, evolving slowly. Further, grain growth is suppressed completely during the decomposition process.
\end{abstract}

Keywords. Spinodal decomposition; grain boundary effects; phase field models.

\section{Introduction}

With the advent of techniques capable of producing metastable crystalline phases with a grain size in the range of $10-100 \mathrm{~nm}$ (examples of such processes would include rapid solidification, laser processing and mechanical milling), there is considerable interest in phase transformations in such materials. In particular, if the metastable alloy has a homogeneous composition which is unstable with respect to phase separation by spinodal decomposition (SD), the characteristic wavelength of SD process is also in the size range of $10-100 \mathrm{~nm}$. Thus, one might expect interesting effects due to the fine grain size on the SD process; this study addresses these effects.

While our emphasis in this study is on fine grained polycrystalline materials, several studies have been reported recently in a related field: surface assisted SD. During the initial stages, a surface layer is formed of that phase for which the surface energy is lower. With increasing distance from the surface, one observes alternating layers of the two phases; deep in the interior, a microstructure similar to that produced by 'normal' (i.e. bulk) SD is observed (see Puri and Binder 1992, 1993, 1994; Fischer et al 1997; Puri et al 1997; Bastea et al 2001). A wide variety of models and simulation techniques have been employed in these studies. Experiments on polymers also reveal a surface wetting layer coexisting with interior, spinodally decomposed two-phase microstructure (Geoghegan et al 2000).

\footnotetext{
*Author for correspondence
}

In our study of phase separation in polycrystalline alloys, we have employed a phase field model, which is a modified version of that used by Fan and Chen (1997) in their study of grain growth in two-phase materials. The essential features of this model are described briefly in the next section. The key results from our study of SD process in a fine grained alloy are presented in $\$ 3$, followed by the main conclusions of this study in $\S 4$.

\section{Formulation}

We use a phase field model similar to the one employed by Fan and Chen (1997). In such phase field models, the variables needed are: $c$, the composition field, and $\boldsymbol{\eta}$ $(i=1,2, \ldots n)$ the 'order parameter' fields which represent the $n$ different orientations of the grains present in the microstructure. The $\eta$ variables are continuum analogs of the orientation variables used in the Potts model (Fan and Chen 1997). $\eta_{\boldsymbol{i}}$ takes an equilibrium value (taken to be unity) within the $i$ th grain and drops to zero outside it, through a grain boundary region where it varies smoothly.

The free energy used in the model is a function of composition, $c$ and the orientation order parameters, $\boldsymbol{\eta}$. We use the rescaled composition variable, $c$, defined by

$$
c=\frac{c^{\prime}-c_{\alpha}}{c_{\beta}-c_{\alpha}},
$$

where $c^{\prime}$ is the composition expressed in mole fraction of species $\mathrm{B}, c_{\alpha}$ and $c_{\beta}$ are the equilibrium compositions of the $\alpha$ and $\beta$ phases, respectively. 
The equilibrium states at low temperature are defined by composition $c=0$ and $c=1$ and $\eta_{\boldsymbol{i}}=1$ and $\eta_{\mathbf{j} \neq i}=0$, $(i=1,2, \ldots n)$. We have chosen the following form for $f\left(c, \eta_{i}\right)$, the bulk free energy density, so that it exhibits these $2 n$ degenerate equilibrium states

$$
\begin{aligned}
& f\left(c, \boldsymbol{\eta}_{i}\right)=A c^{2}(1-c)^{2}+B(m c+1) \\
& \left\{1+\sum_{i}\left(-\eta_{i}^{2}-2 \eta_{i}^{3}+2 \eta_{i}^{4}\right)+\varepsilon \sum_{i} \sum_{j \neq i} \eta_{i}^{2} \eta_{j}^{2}\right\},
\end{aligned}
$$

where $A, B$ and $m$ are phenomenological constants. The constant, $m$, couples the composition, $c$, and the orientation order parameters, $\boldsymbol{\eta}$, such that the energy of the grain boundary formed in the $\alpha$ phase $\left(\gamma_{\mathrm{gb}}^{\alpha}\right)$ is lower than that formed in the $\beta$ phase $\left(\gamma_{\mathrm{gb}}^{\beta}\right)$. This model can account for the following different types of interfaces at low temperatures: grain boundary in $\alpha$ and $\beta$-phases, coherent interface (within the same grain) and incoherent interface (across two different grains) between $\alpha$ and $\beta$ phases.

The total free energy, $F$, of an inhomogeneous system is given by

$$
F=\int_{V}\left[f_{0}\left(c, \boldsymbol{\eta}_{\mathbf{i}}\right)+\frac{\kappa_{c}}{2}(\nabla c)^{2}+\sum_{i} \frac{\kappa_{i}}{2}\left(\nabla \boldsymbol{\eta}_{i}\right)^{2}\right] \mathrm{d} V,
$$

where $\kappa_{\varepsilon}$ and $\kappa_{i}$ are the gradient energy coefficients associated with inhomogeneities in composition and in $\boldsymbol{\eta}$, respectively.

The evolution of the composition is governed by Cahn-Hilliard equation

$$
\frac{\mathrm{d} c}{\mathrm{~d} t}=M \nabla \cdot\left(\nabla\left(\frac{\delta F}{\delta c}\right)\right)
$$

where $M$ is the mobility, assumed to be independent of composition. On the other hand, the evolution of the $\eta$ variables is governed by Cahn-Allen equation

$$
\frac{\mathrm{d} \eta_{i}}{\mathrm{~d} t}=-L_{i} \frac{\delta F}{\delta \eta_{i}}
$$

where $L_{i}$ are the relaxation parameters.

\section{Results}

We have used a control volume method (see Patankar 1980) for solving the above evolution equations in two dimensions. The simulation is carried out on a $256 \times 256$ square grid with $\Delta x=\Delta y=1 \cdot 0$. For time integration, we used a time interval of $\Delta t=0 \cdot 1$. Our simulation has used the following values of the model parameters: $A=1.0$, $B=0.5, \quad m=1 \cdot 0, \quad L_{i}=1 \cdot 0, \quad M=1 \cdot 0$. The above equations are given in their dimensionless forms; the characteristic energy, length and time used for this scaling procedure are chosen in such a way that the parameters $A, M$ and $\kappa_{\varepsilon}$ are of order unity. The simulation starts with an alloy with a composition of $c=0.5$, on which we superimpose an initial (uniform) random noise of 0.01 at the grid points.

\subsection{Spinodal decomposition in a single crystal}

Figure 1 shows the evolution of spinodally decomposed microstructure in a single grain after 100, 500, 1000 and 2000 time steps. During early stages, it is clear that the initial fluctuations have grown in amplitude. Those fluctuations with a wavelength of

$$
\lambda_{\max }=4 \pi\left[-\kappa /\left(\partial^{2} f / \partial c^{2}\right)\right]^{1 / 2},
$$

grow the fastest (Cahn 1961); therefore, in the microstructure after, say, 100 time steps, the typical distance between two neighbouring white features (B-rich regions) is about $\lambda_{\text {max }} \approx 17$. The $\mathrm{A}$-rich and $\mathrm{B}$-rich regions form a bicontinuous network which, beyond 1000 steps, undergo coarsening. Further, only after about 1000 steps, the composition of the A-rich and B-rich regions reach the equilibrium values of $c=0$ and $c=1$, respectively.

\subsection{Grain boundary assisted spinodal decomposition}

For this simulation, an initial polycrystalline microstructure is needed. Such a microstructure was obtained using a prior simulation, which started with an initial configuration with $\boldsymbol{\eta}_{\mathbf{n}}=0+\boldsymbol{\delta} \boldsymbol{\eta}$ (where $\boldsymbol{\delta} \boldsymbol{\eta}$ is a small positive noise); the integration of Cahn-Allen equation was carried out up to 3000 time steps, and it produced a microstructure with grain boundaries (see figure 2(a) in which the grain boundaries are decorated by being enriched with species A). The spinodal decomposition in a polycrystalline alloy was simulated using this microstructure, by adding a homogeneous composition of $c=0.5$ (with a noise of 0.01) to this configuration. Figure 2 shows the sequence of microstructures of a polycrystalline system after 100, 500, 1000 and 2000 time steps.

The very early stages of decomposition are characterized by enrichment of the grain boundaries by the species A; this can be seen in the figure as early as $\tau=100$ time steps. As time progresses, the grain boundaries become decorated with the A-rich $\alpha$ phase, with the B-rich $\beta$ phase on either side of it. This process can be thought of as one in which the A-enrichment triggers a composition wave normal to the grain boundary, which travels into the grain interior. By 500 time steps, it is clear that the composition of the A-rich phase at the grain boundary has reached its equilibrium value of $c=0$. This may be compared with that in single crystal, wherein the A-rich phase composition is only about $0 \cdot 3$ after 500 steps. Thus, it is clear that the GB-initiated spinodal decomposition occurs much faster than the normal one.

While the GB-initiated composition wave propagates into the grains, normal spinodal decomposition could- 

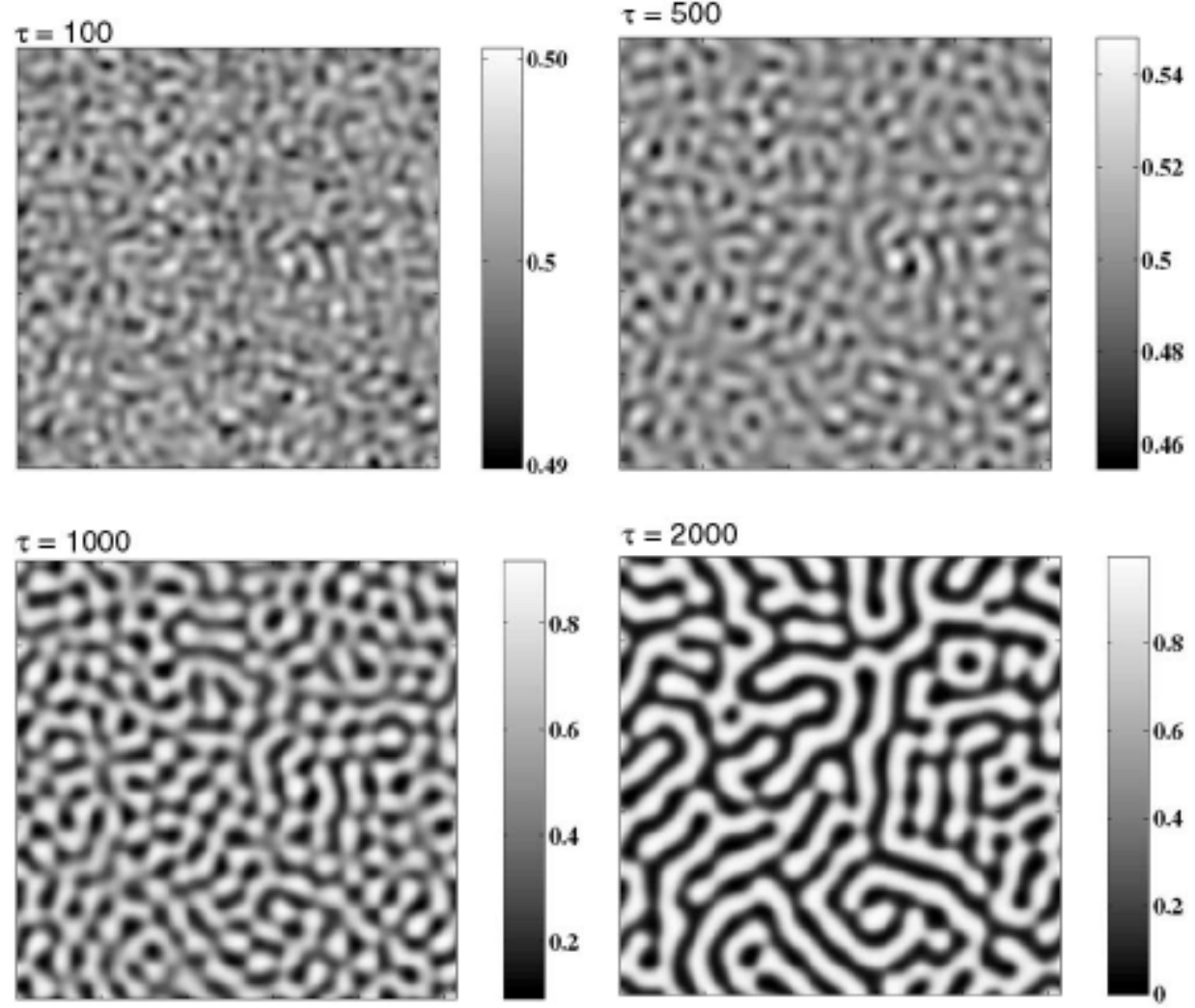

Figure 1. The evolution of SD microstructure in a single crystal after $\tau=100,500,1000$ and 2000 time steps.
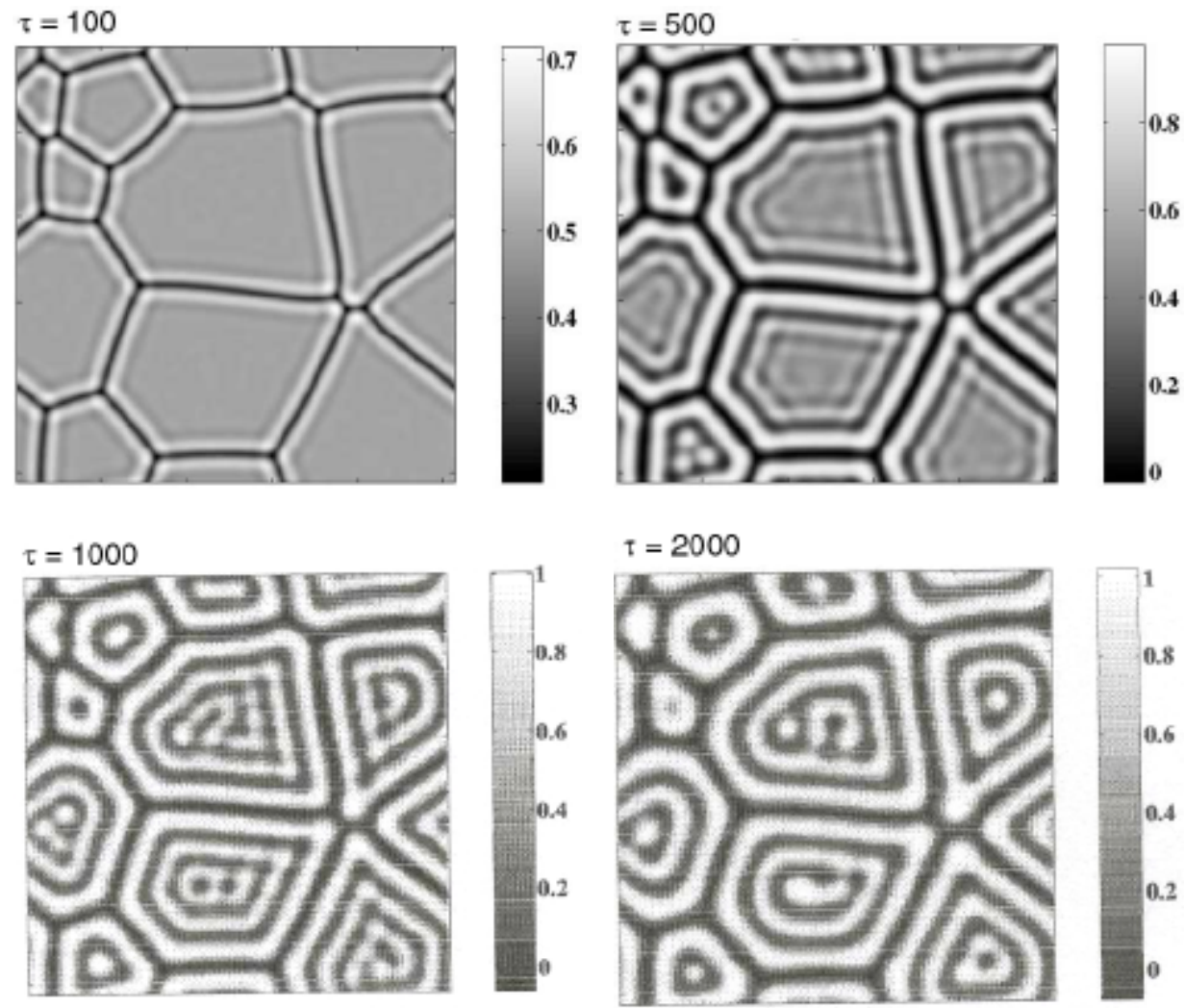

Figure 2. The evolution of SD microstructure in a polycrystalline alloy after $\tau=100,500$, 1000 and 2000 time steps. 
and indeed does - take place deep in the grain interiors (see the top right regions in figure 2 after 1000 steps). Thus, the late stage microstructure (say, after 2000 time steps) shows a mixture of GB initiated composition waves co-existing with the usual spinodal waves in the grain interiors of large grains. In small grains, however, the GB-initiated waves travel fast enough to cover the entire grain, before normal spinodal decomposition occurs in the interior; in these grains, the dominant features are concentric alternating rings of A-rich and B-rich phases. During this entire course of events, the grain boundaries are virtually immobile; since the grain boundaries are covered by the A-rich phase, any movement on their part normal to themselves will take them through a configuration in which they lie between A-rich and B-rich regions; in other words, such a movement leads to converting an $\alpha$ phase grain boundary into an incoherent interface with a much higher energy, thus rendering the process of grain growth virtually impossible.

Figure 3 shows the structure function, $S(k)$, during different times both for the single crystal as well as the polycrystalline case. The structure function, $S(k)$ is plotted as a function of $k$ for 100,500 and 2000 time steps. A comparison of the plots for the same time (e.g. 500 time steps) shows that the peak in $S(k)$ in the polycrystalline

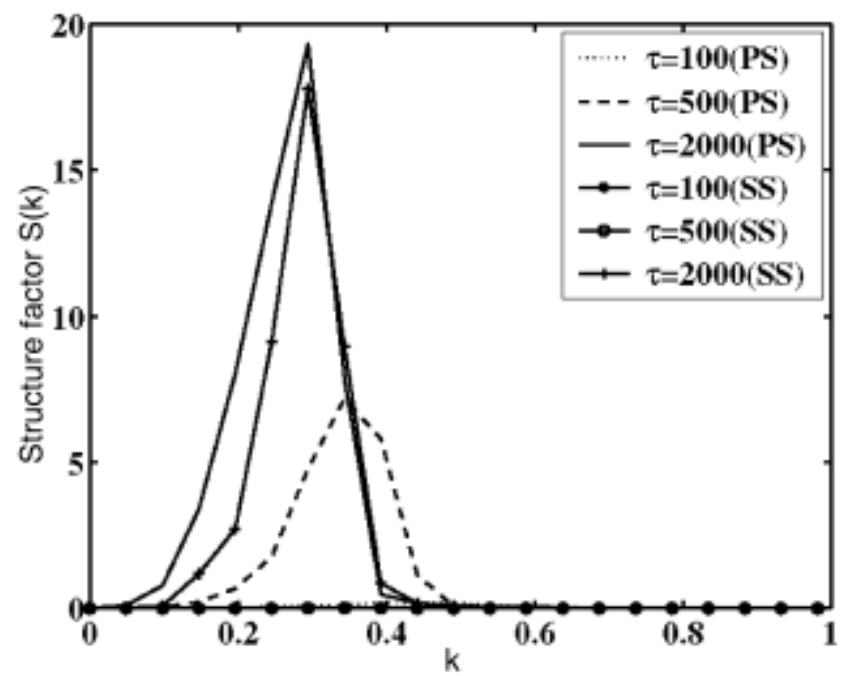

Figure 3. The evolution of the structure factor, $S(k)$ for single crystal (dotted line) and polycrystalline system (continuous line) for $\tau=100,500$ and 2000 time steps. The symbol 'ps' and 'ss' refer to polycrystalline and single crystalline alloys, respectively. case is much higher than that in the single crystal; this indicates that spinodal decomposition has taken place to a larger extent in the former than in the latter. Further, the peak in the polycrystalline case shifts to the left from $k=0.4418$ through 0.3436 to 0.2945 at $\tau=100,500$ and 2000 time steps, respectively. For the single crystal, on the other hand, the peak in $S(k)$ remains at $k=0.3436$ up to $\tau=500$ and shifts to a lower value of $k=0.2945$ at $\tau=2000$ time steps. This is clearly due to the influence of the grain size (which is several times larger than the characteristic spinodal wavelength).

\section{Conclusions}

We have studied the spinodal decomposition process in a polycrystalline alloy with a grain size, which is similar to the dominant decomposition wavelength. When the grain boundary energy, $\gamma_{\mathrm{gb}}^{\alpha}$, of A-rich $\alpha$ phase is lower than that of the B-rich $\beta$ phase, the decomposition process is initiated with the formation of $\alpha$ layer at the grain boundaries. In small grains, the interior of the grain is filled with the $\beta$ phase, while in large grains, the interior exhibits the usual spinodal microstructure. The kinetics in a polycrystalline alloy is much faster than that in a single crystal. Further, during the phase separation process, the grain growth is completely suppressed.

\section{Acknowledgements}

We thank the Council of Scientific and Industrial Research, New Delhi, for financial support.

\section{References}

Bastea S, Puri S and Lebowitz J L 2001 Phys. Rev. E63 041513 Cahn J W 1961 Acta Metall. 9795

Fan D and Chen L-Q 1997 Acta Mater. 453297

Fischer H P, Maass P and Dieterich W 1997 Phys. Rev. Lett. 79 893

Geoghegan M, Ermer H, Jungst G, Krausch G and Brenn R 2000 Phys. Rev. E62 940

Patankar S 1980 Numerical heat transfer and fluid flow (New York: Hemisphere Publishing Corporation, McGraw-Hill Book Company)

Puri S and Binder K 1992 Z. Phys. B86 263

Puri S and Binder K 1993 Phys. Rev. E49 535

Puri S and Binder K 1994 J. Stat. Phys. 77145

Puri S, Binder K and Frisch H L 1997 Phys. Rev. E56 6991 